\title{
The effect of the observer vantage point on perceived distortions in linear perspective images
}

\author{
DEJAN TODOROVIĆ \\ University of Belgrade, Belgrade, Serbia
}

\begin{abstract}
Some features of linear perspective images may look distorted. Such distortions appear in two drawings by Jan Vredeman de Vries involving perceived elliptical, instead of circular, pillars and tilted, instead of upright, columns. Distortions may be due to factors intrinsic to the images, such as violations of the so-called Perkins's laws, or factors extrinsic to them, such as observing the images from positions different from their center of projection. When the correct projection centers for the two drawings were reconstructed, it was found that they were very close to the images and, therefore, practically unattainable in normal observation. In two experiments, enlarged versions of images were used as stimuli, making the positions of the projection centers attainable for observers. When observed from the correct positions, the perceived distortions disappeared or were greatly diminished. Distortions perceived from other positions were smaller than would be predicted by geometrical analyses, possibly due to flatness cues in the images. The results are relevant for the practical purposes of creating faithful impressions of 3-D spaces using 2-D images.
\end{abstract}

Almost from the inception of linear perspective as a system of representing spatial layouts, it was noted that some perspective images whose construction is geometrically correct may still look deviant in certain respects. These effects are often referred to as marginal distortions, because they usually appear toward the sides of paintings and drawings (Kubovy, 1986). For example, in perspective images of ground planes covered with square tiles, often seen in Renaissance paintings, the tiles positioned toward the left and right edges of the scene may look not square but elongated and skewed. In the 15th century, the painter-geometer Piero della Francesca studied the conditions under which such effects are manifested (Field, 1986), and the problem is still under investigation (Juricevic \& Kennedy, 2006). Under similar conditions, correct projections of cubes may appear as definitely not cube-like (Olmer, 1943/1949). In the painting School of Athens, Raphael drew off-center spheres as circles rather than as ellipses, although this is the proper shape for their perspective image, possibly in order to avoid their looking distorted (Pirenne, 1970). Perspective images of colonnades of columns running parallel to the projection plane have increasingly thinner angular projections with increasing distance but, paradoxically, increasingly wider linear perspective projections. Leonardo da Vinci was apparently quite intrigued by this particular perspective effect, since he drew about a dozen geometrical sketches of this situation (see Veltman, 1986). In this article, I will examine in detail two examples of the distorted appearance of linear perspective, using drawings by Jan Vredeman de Vries
(1604/1970), and will report two experiments in which the cause of the distortions was studied.

Observing Figure 1 (I have added the circled numerals 1 through 5 next to some columns), it is easy to agree with Kubovy (1998) that "something must have gone wrong with this depiction" (p. 482). Did de Vries really intend to depict a bizarre building with diverging columns that subtend various oblique angles with respect to their base surface? Or did he make a mistake in the perspective construction? If, as is likely, he had a conventional architectural structure in mind and the construction is geometrically correct, why does it look so distorted?

Another puzzling, although less dramatic, example can be seen in another drawing by de Vries (see Figure 2; I have added the circled letters $\mathrm{A}, \mathrm{B}_{1}, \mathrm{~B}_{2}$, $\mathrm{d}$, and $\mathrm{h}$ ). Note that the oval pillars at the left do not look round but, rather, look elliptically elongated into depth. Did the artist actually attempt to represent elliptical pillarsobjects rarely, if ever, used in real architecture? Did he perhaps deliberately intend these two figures to provide demonstrations of perceptual distortions, or was it simply a matter of inattention to details? ${ }^{1}$ Or do these examples provide more evidence that linear perspective is an inadequate system of representing space, as some have argued (Goodman, 1976)?

The answers to the questions above are of interest beyond explaining the curious looks of these particular two figures. They address central issues of pictorial perception, involving the fundamental question as to how well 2-D images are able represent 3-D scenes and the corresponding applied question as to how 2-D images should

D. Todorović, dtodorov@f.bg.ac.rs 


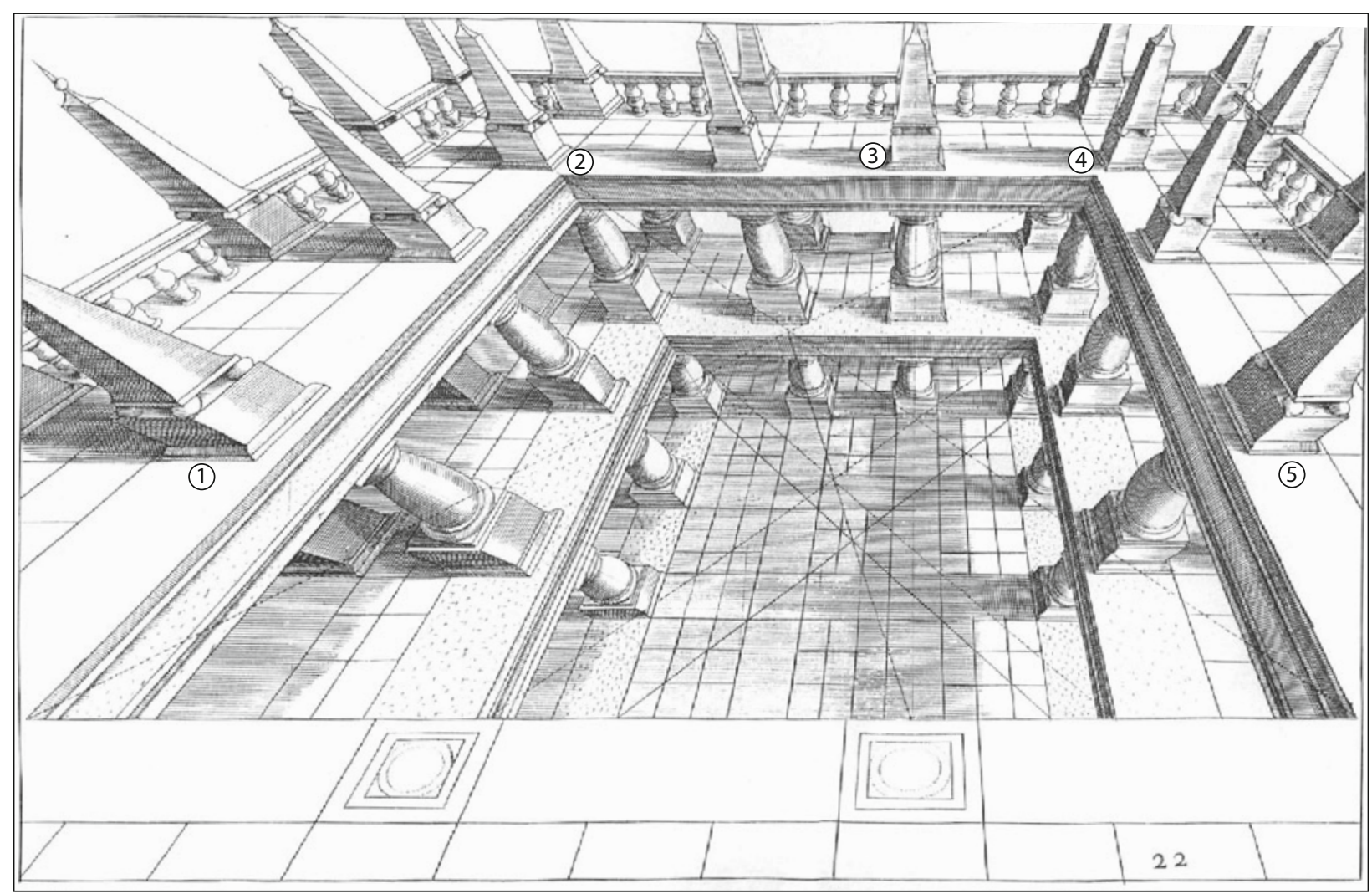

Figure 1. An engraving by de Vries (1604/1970). Note that some columns appear to have an oblique, rather than orthogonal, orientation with respect to the terraces, especially those at the left-hand side on the top terrace. From Perspective, by Jan Vredeman de Vries, 1604/1968, New York: Dover. Copyright 1968 by Dover Publications. Reprinted with permission.

be constructed and displayed in order to depict 3-D scenes as adequately as possible.

There are two potential sources of perceived distortions in correctly constructed linear perspective images and, thus, two main, mutually nonexclusive explanatory strategies to account for such effects. One strategy is intrinsic, and it invokes the features of the content or the structure of the images, such as the types of depicted bodies or the aspects of perspective projections, that may potentially lead to their distorted appearance. The other is extrinsic, and it proposes that such effects may arise as a consequence of certain conditions of observation, including the spatial location of the observer with respect to the image, and the orientation of the image with respect to the observer.

An example of the intrinsic strategy is Kubovy's (1986) statement that "certain geometrically correct central projections give rise to pictures that violate Perkins's laws and therefore do not look right" (p. 100). Perkins's laws are empirical rules whose aim is to provide constraints on perceptual interpretations of images of trihedral corners of cuboids (see Kubovy, 1986; Perkins, 1973; Shepard, 1981). Such corners can project into fork junctures and arrow junctures. The laws state that such 2-D junctures will be perceived to represent 3-D constellations of a cube's corner (three right angles) only if they fulfill certain conditions: For fork junctures, none of the three angles may be smaller than $90^{\circ}$, and for arrow junctures, none of the two angles may exceed $90^{\circ}$ but their sum must exceed $90^{\circ}$. Kubovy (1998) showed that the depiction of the base of Pillar 1 in Figure 1 violates both of Perkins's laws. Thus, one account of the unusual look of this figure is that even though it may be a correct drawing of a conventional construction, it does not look correct, because it does not comply with Perkins's laws.

An example of the extrinsic strategy is to account for the distorted appearances as being due to observers' inappropriate choices of vantage points. As is well known, every linear perspective image has associated with it a projection center, and only when observed from that position does it provide essentially the same stimulus that the corresponding 3-D scene would provide. If the vantage point does not coincide with the projection center, the stimulus provided by the image deviates from the original stimulus. Geometrical analyses dating from the 19th century on have indicated that displacements of the vantage point with respect to the image surface are associated with corresponding deviations of the 3-D layout conveyed by the image. Thus, displacements orthogonal to the image surface correspond to compressions (for motions toward the image) and dilations (for motions away from the image) of the conveyed scene, whereas displacements parallel to the image surface correspond to lateral shears of the conveyed scene, in the direction opposite to the motion of the observer (see Cutting, 1988, 1993; de la Gourn- 


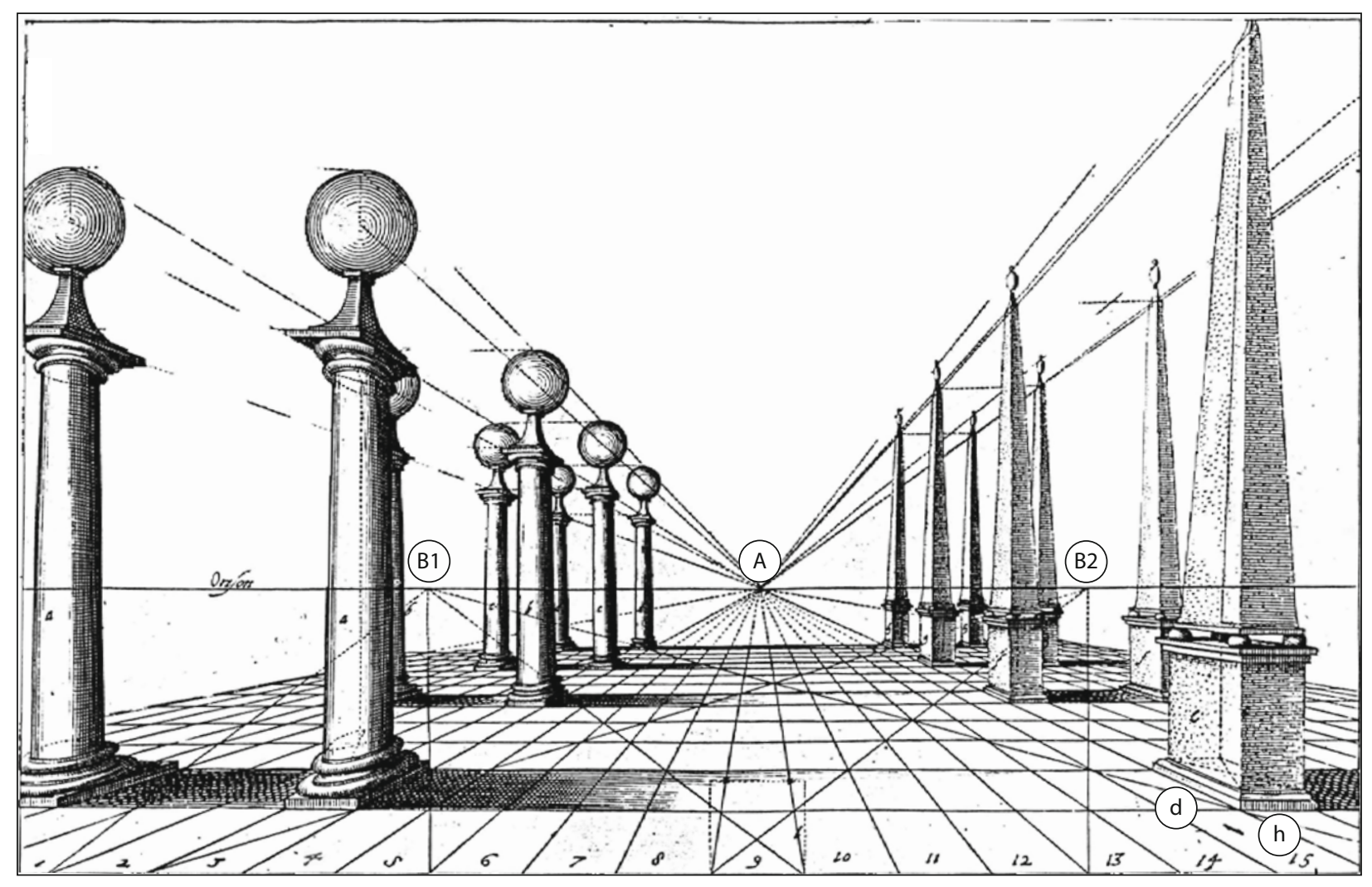

Figure 2. An engraving by de Vries (1604/1970). Note that the oval pillars on the left-hand side appear to be elliptical, rather than circular. From Perspective, by Jan Vredeman de Vries, 1604/1968, New York: Dover. Copyright 1968 by Dover Publications. Reprinted with permission.

erie, 1859; Kubovy, 1986; Nicholls \& Kennedy, 1993; Sedgwick, 1993; ten Doesschate, 1964; Todorović, 2005, 2008). These geometrical analyses suggest that the effects in Figures 1 and 2 may be due to the deviation of the vantage points of the observers from the appropriate perspective projection centers of the images. If de Vries's constructions are correct and depict architectonically conventional structures, the vantage point hypothesis predicts that if the images were to be observed from their proper vantage points, the distortions would mostly disappear: Under such conditions, in spite of violating Perkins's laws, the columns in Figure 1 should not appear oblique but should appear perpendicular to the terrace, and the pillars in Figure 2 should look not elliptical but circular.

In support of this hypothesis, Pirenne (1970) found that certain photographs created with a pinhole camera cease to look distorted when observed from the position of the projection center. On the other hand, commenting on Raphael's spheres, Perkins (1973) argued that even observation from the proper vantage point may not suffice to induce the appropriate percept for some figures. Furthermore, a number of authors have favored the notion that the transformations predicted by the geometrical analyses may, in fact, generally not be perceptually realized. The idea is that, except in special circumstances, a hypothesized compensation process would recover the correct projection center and would reconstruct the appropriate layout even when the image is observed from inappropriate vantage points (Goldstein, 2001; Haber, 1980; Pirenne, 1970; Shepard, 1992; see also the considerations by Kubovy, 1986, and Yang \& Kubovy, 1999, who proposed a modified compensation hypothesis). If this is true, the position of the vantage point should, in general, not significantly affect the perception of virtual space in pictures.

A number of empirical studies, reviewed by Rogers (1995), have shown that displacements of the vantage point, on the whole, do tend to induce perceptual changes similar to the transformations predicted by geometrical analyses, but with wide variations in the strengths of such effects in different studies. The results of more recent studies are also somewhat discrepant. Vishwanath, Girshick, and Banks (2005) reported that manipulations of the position of the vantage point had relatively small effects on the perceived geometrical structure of depicted bodies, especially for modest displacements of the vantage point, whereas Todorović (2008) found relatively strong effects on perceived 3-D directions. Juricevic and Kennedy (2006) reported effects of vantage point variations that were often smaller than those predicted by the geometrical analysis; furthermore, even when observed from the correct vantage points, many shapes in the images looked distorted. One difference between these three studies was that Todorović (2008) used vantage point displacements parallel to the image surface, Juricevic and Kennedy used 
orthogonal displacements, and Vishwanath et al. used displacements positioned effectively along a semicircular path, which can be represented as a combination of parallel and orthogonal displacements. A general problem with the existing literature on this topic is that different studies have used rather different manners of vantage point manipulations, presentation conditions, types of images, and judgments required from observers, as well as response measures, so that it is not easy to compare them and generalize the results. Here, I report two experiments in which Figures 1 and 2 were used as stimuli to study the cause of the perceived distortions in these drawings. Because the perspective structure of Figure 2 is simpler, the corresponding experiment will be reported first.

\section{EXPERIMENT 1}

To test the conjecture that the unusual appearance of portions of Figure 2 could be due to the inadequate location of the vantage point of the observer, the appropriate position of the projection center for this figure must be determined. In some previous studies of the role of the vantage point (Juricevic \& Kennedy, 2006; Todorović, 2008; Vishwanath et al., 2005), the authors themselves constructed the stimuli used in the research and, thus, were in a position to control and manipulate their projection centers. In other studies, the authors used already existing artworks as stimuli and manipulated the locations from which they were observed (Deregowski \& Parker, 1992; Goldstein, 1979; Halloran, 1993; Wallach, 1976) but did not determine their projection centers. Reconstructing the projection center of a given image is a problem that has been studied extensively in the literature (see Green, 1983; Kemp, 1990; Sedgwick, 1980) and is still actively researched (Halloran, 2006). The feasibility of these techniques depends on some properties of images and assumptions about the depicted layout. They were used by art experts to recover the positions of projection centers of some well-known paintings (Dubery \& Willats, 1983; Field, 1997; Wittkower \& Carter, 1953) but have apparently not been applied for artworks used in experimental studies of vantage point effects. In the present study, I used these techniques to recover the projection centers of the de Vries drawings.

Figure 2 is a one-point perspective construction, with a single principal vanishing point, denoted by A. Such images often depict a ground plane ruled by a square grid. The projection of a square grid is simple to construct and involves basic perspective rules dating from the 15 th and 16 th centuries, so that it is reasonable to assume that de Vries had such a layout in mind. The diagonal lines of the grid have their own vanishing points, denoted by $B_{1}$ and $\mathrm{B}_{2}$, which are positioned at the same distance from $\mathrm{A}$, at opposite sides. In images with such features, the reconstruction of the projection center follows a standard procedure. Namely, it can be shown that the projection center is located on a line that is perpendicular to the image surface and passes through the principal vanishing point and that its distance from the image is equal to the distance between the principal vanishing point and either of the two diagonal vanishing points, which are called distance points for this reason (Green, 1983; Kemp, 1990; Sedgwick, 1980).
As can be seen by inspecting the image, the distance from $\mathrm{B}_{1}$ or $\mathrm{B}_{2}$ to $\mathrm{A}$ and, thus, the appropriate observation distance for Figure 2 amounts to about one quarter of the width of the figure itself. This fact leads to an important conclusion: Given the size of the reproduction of the figure in this article and generally in art books, this is far too small an extent for most observers. From this it follows that this figure is usually observed from distances larger than the distance of its projection center. As was noted above, geometrical analyses indicate that increasing the observation distance is associated with dilation of the virtual space conveyed by perspective images. In particular, such observation distances should induce perceptual elongations into depth of the pillars depicted in the image, making them appear elliptical, as is indeed the case. Thus, the reconstruction above of the projection center of Figure 2 corroborates the idea that the perceived distortions are due to the inadequate location of the vantage point of observers.

Since the distance of the projection center is proportional to the size of the image, by enlarging the image this distance can be increased and made more accessible to observers. Informal observations using enlarged copies of Figure 2 revealed that varying the distance from the figure resulted in surprisingly salient changes in the apparent depth dimensions of the represented objects. In particular, in close-up view, the oval columns did look approximately round, rather than elongated into depth. Furthermore, the horizontal cross-sections of the pyramid-bearing cuboid bases of the columns on the right-hand side of the drawing, which in the reproduced figure look distinctly like rectangles elongated into depth, appeared more square-like in a close-up view of the enlarged image; this was in accord with the fact that the column base covered exactly one tile of the ground plane perspective grid, which, by hypothesis, was constituted by depicted squares. The informal observations on the column bases were tested in a formal experiment. Note that the projections of their trihedral corners do conform to Perkins's laws. Thus, a theory of perceived distortions based on these laws does not predict that they should look distorted. On the other hand, the vantage point position hypothesis, which claims that perceived dimensions of image features depend on the location of the vantage point, predicts that increasing the observation distances from the image should be associated with increases in the perceived depth extents of the column bases.

\section{Method}

Subjects. The subjects were 12 first-year University of Belgrade psychology students, who served for course credit.

Stimulus and Setup. The drawing, without the circled letters, was printed on a paper with the width $\times$ height dimensions of $111 \times$ $73 \mathrm{~cm}$ and was hung on the wall of the experimental room, with the bottom edge positioned $139 \mathrm{~cm}$ above the ground. The distance between the vanishing point and either one of the distance points, which specified the predicted correct distance of the vantage point, was $28 \mathrm{~cm}$. On the floor of the room, there was a long, thin plank oriented perpendicularly to the wall containing the image, with one end positioned directly below the vanishing point.

Procedure. At the start of the experimental session, the subjects were taken for a short walk along the plank toward the display, starting from a distance of about 6-7 $\mathrm{m}$ from it. The subjects' attention was directed toward the base of the rightmost column in the front 
row. They were asked whether there was an apparent change in its dimensions, without suggesting its direction. All the subjects reported that the base appeared to shrink in depth as they walked toward it. They were then asked to judge the dimension of the depth-directed side of the base (denoted as "d" in Figure 2), as compared with its horizontal side (denoted as "h"), as follows. They were asked to walk along the plank, keeping their left foot at its left side and their right foot at its right side. Their task was to move back and forth in this way and stop at the distances from the display from which the depthdirected side of the base looked (1) just as long as the horizontal side (the " $1: 1$ " task), (2) one-and-a-half times as long (the "1.5:1" task), and (3) twice as long (the " $2: 1$ " task). It was stressed in the instruction that the judgments were to refer to the dimensions in the depicted 3-D scene, rather than to the lines on the printed 2-D image. The distances were read off a measuring tape affixed to the side of the plank. They were measured from the back of the heels of the observers' shoes to the wall with the image. Since there were three judgments to be made, there were six possible orderings, and each one was used for 2 of the 12 subjects. This technique, in which the image was stably anchored in its 3-D surrounding, was an inverse of the procedure used by Nicholls and Kennedy (1993), where the observer was stationary and the image was moved.

\section{Results and Discussion}

The results are presented in Table 1 . As the required perceived depth-side/horizontal-side ratio of the column base increased, the corresponding observation distance increased as well. A repeated measure one-way ANOVA yielded a significant effect $\left[F(2,22)=159.75, M S_{\mathrm{e}}=\right.$ 485,592, $p<.000001]$.

There are three main conclusions from the data. First, there was a clear effect of observation distance, in agreement with the vantage point position hypothesis: Viewed from increasingly larger distances, the column base looked increasingly more elongated into depth. Second, according to geometrical analyses, the column base should have looked square, when observed from the proper projection center, at the distance of $28 \mathrm{~cm}$. The obtained mean distance from which the depth side looked equal to the horizontal side was $21.38 \mathrm{~cm}$, which was not significantly different from the prediction $[t(11)=1.37, p>.19]$. Thus, when the image was observed from the reconstructed geometrically appropriate location, its appearance was in accord with what one may suppose were the original intentions of the artistthat is, to depict a conventional architectonic scene. Third, although the elongation effect was in the direction predicted by geometrical analyses, quantitatively it was much weaker. Geometrical analyses indicate that the extent of dilation should be proportional to the increase of the distance from the image. Thus, given that the depth-side/horizontal-side ratio was equal to 1:1 when the image was observed from the theoretically predicted distance of $28 \mathrm{~cm}$, the ordained ratio of 1.5:1 should have been obtained for an observation

Table 1

Mean Predicted and Obtained Distances and Standard Deviations for the Three Types of Tasks in Experiment 1

\begin{tabular}{cccc}
\hline $\begin{array}{c}\text { Task } \\
\text { Required Depth/ } \\
\text { Horizontal Side Ratio) }\end{array}$ & $\begin{array}{c}\text { Predicted } \\
\text { Distance } \\
(\mathrm{cm})\end{array}$ & $\begin{array}{c}\text { Obtained } \\
\text { Distance } \\
(\mathrm{cm})\end{array}$ & $\begin{array}{c}\text { Standard } \\
\text { Deviation } \\
(\mathrm{cm})\end{array}$ \\
\hline $1: 1$ & 28 & 21.4 & 16.7 \\
$1.5: 1$ & 42 & 252.9 & 52.7 \\
$2: 1$ & 56 & 422.1 & 92.3 \\
\hline
\end{tabular}

distance of $42 \mathrm{~cm}$, but it was actually obtained at a distance about 6 times larger; similarly, the ratio of 2:1 should have been obtained for a distance of $56 \mathrm{~cm}$, but it was obtained at a distance about 7.5 times larger. This issue will be taken up in the General Discussion section.

One potential source of bias in the obtained results is the fact that the distance of the observers from the image was measured from the heels of their shoes, rather than from their eyes. Given the conditions of the study and the nature of the task, in which the observers selected the appropriate distance themselves, this was a practically convenient measure. However, for a person standing upright and holding his or her head still, the position of the eyes in space is in front of the back of the heels. For the 1.5:1 and $2: 1$ task, this measure thus tended to overestimate the distance of the eyes to the image, although probably only for a modest percentage, relative to the sizes of the recorded distances. However, for the 1:1 task, in which the subjects were standing very close to the picture, the direction of the bias was harder to estimate, because the subjects generally did not hold their heads perfectly upright, occasionally leaned back, and also tended to turn their heads toward the right side, to fixate the to-be-judged base of the depicted column. Although these sources of bias and variability decrease the reliability of the quantitative aspects of the results to some extent, they do not challenge the robustness of the main findings, which are that increasing the observer distance increased perceived pictorial depth; that for the 1:1 task, the recorded mean distance was reasonably close to the prediction; and that for the 1.5:1 and 2:1 tasks, the sizes of the recorded mean distances were in the predicted direction and order, although much larger.

The results of Experiment 1 are in accord with the findings of Juricevic and Kennedy (2006). They found that in a perspective image of a ruled ground plane tiled with squares, many quadrilaterals that were perceived, correctly, as squares when viewed from a smaller distance looked elongated when viewed from a larger distance. The authors presented a theory that predicts the perceived shapes of such figures on the basis of the ratio of their sides and the angle that their direction with respect to the observer subtends with the direction normal to the image surface. The specific values of the parameters of the predictive equations depend on the content of the image, so that it was not possible to use the theory to quantitatively predict the outcome of the present study and, in particular, to predict whether the target stimulus would or would not be perceived as distorted when observed from the correct position. Furthermore, the saliency of pictorial depth conveyed by the stimuli used in the two studies was different, since Juricevic and Kennedy used bare ruled ground planes lacking any objects, whereas the de Vries drawing is an artistic rendering that depicts not just a plane but a 3-D scene with columns extending into depth.

\section{EXPERIMENT 2}

This experiment assessed the perceived distortions in Figure 1. As in the case of Figure 2, the reconstruction of the projection center in Figure 1 depends on some as- 
sumptions about the depicted layout and properties of the image. I have assumed that de Vries had in mind an ordinary architectural construction with horizontal terraces and vertical pillars. Whereas Figure 2 was executed in one-point perspective, a construction that was used in a large number of paintings and drawings from the early Renaissance on, Figure 1 involves two-point perspective, which is a more complex construction. Usual examples of such constructions involve a house depicted at an oblique orientation, with a prominent vertical near edge shared by two sides of the house; neither of the two sides is oriented frontally, and they have different vanishing points, both of which are located on the horizon. However, Figure 1 is a more rare variant in which the two principal vanishing points, one for the pillars and the other for the depthdirected sides of the terrace, do not lie on a horizontal line but, rather, on a vertical line. It can be produced by projecting the to-be-depicted object upon a tilted projection plane $\Pi$, such as sketched in Figure 3A.

The standard procedure for reconstructing the projection center of two-point perspective images starts with the construction of a circle that contains the two vanishing points $\mathrm{V}_{1}$ and $\mathrm{V}_{2}$, with the diameter equal to the distance between

A

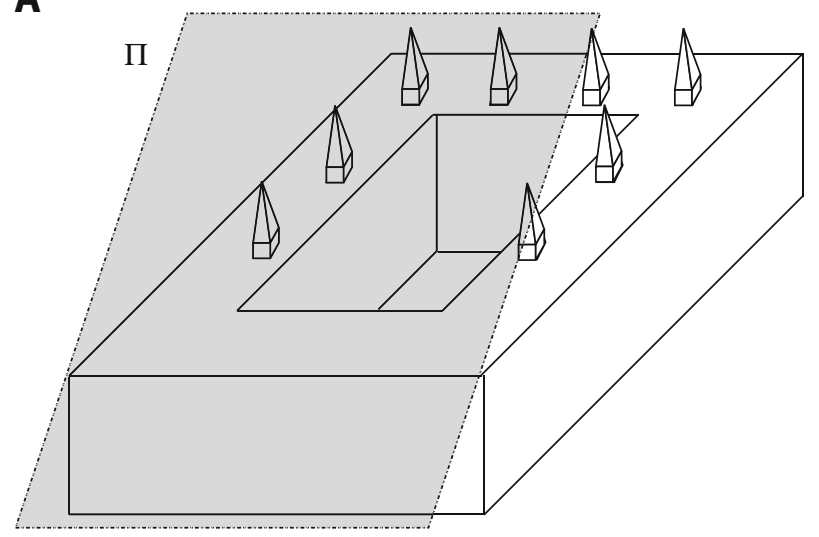

B

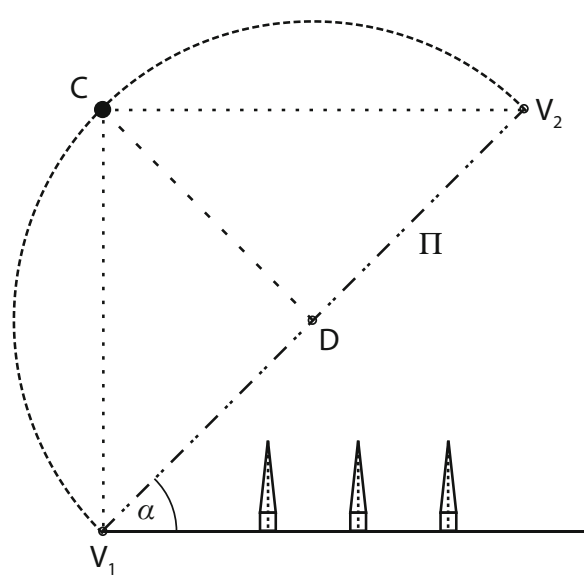

Figure 3. (A) Parallel perspective sketch of a possible setup of the scene depicted in Figure 1, involving a conventional architectural construction projected upon a tilted projection plane. (B) Side view. See the text for details. them, as depicted in Figure 4. It can be shown that the projection center must belong to such a circle, which lies in a plane perpendicular to the projection plane, as depicted in side view in Figure 3B (see Green, 1983; Sedgwick, 1980), because choosing any point on such a circle as the projection center ensures, by Thales's theorem, that two main sets of directions, such as those defined by columns and by terrace sides, are mutually perpendicular. The next step consists in identifying a particular point on that circle as the correct projection center. The standard way to do this involves constructing vanishing points of diagonals of certain components in the image that are known to be square in the scene and to be positioned in certain orientations and that also have to be relatively large in order for the geometric reconstruction to be acceptably accurate (for details, see Sedgwick, 1980). However, it turns out that such features cannot be reliably located in Figure 1, so the standard approach is hard to apply in this case.

An alternative procedure for reconstructing the location of the projection center for this figure is to hypothesize the value of the angle that the projection plane subtends with the plane of the terrace. The simplest and most symmetrical possibility is that this value is halfway between the side view and the top view of the scene. In this case, it equals $\alpha=45^{\circ}$, as depicted in the sketch in Figure 3B. As was noted above, it is also assumed that the terrace is horizontal and the columns vertical. Given these assumptions, it follows that the projection center $\mathrm{C}$ must be located halfway between the two vanishing points $\mathrm{V}_{1}$ and $\mathrm{V}_{2}$ on the circle, because only in that case do the lines that connect $\mathrm{C}$ to the vanishing points have vertical and horizontal orientations, respectively.

Provided that this construction has correctly recovered the position of the projection center, it follows that its distance from the projection plane $\Pi$ is equal to the radius of the circle (line CD in Figure 3B). An inspection of Figure 4 indicates that this extent amounts to about a third of the width of the figure. Thus, as in Figure 2, for the scale at which Figure 1 is reproduced in this article or in art books, this extent is far too small to be acceptable as a usable observation distance. Note also that if the correct projection center were located anywhere else on the circle, its distance from the image would be even smaller. On the basis of these considerations, it can be concluded that, as in case of Figure 2, Figure 1 is usually observed from distances larger than those prescribed by the perspective construction. ${ }^{2}$

The consequences of displacing the vantage point away from the correct projection center for Figure 1 are illustrated in Figure 5 (see Cutting, 1988, 1993; Sedgwick, 1993; Todorović, 2005, 2008). If the distance CD in Figure 5A is taken as one unit, the sketch in Figure 5B represents the effect of moving the vantage point orthogonally back one more unit: The consequence is a dilation in which each point in the conveyed scene in the virtual space is displaced at twice the original distance from the image plane. Note that the original right angle subtended by the column and the terrace is now distorted; calculations show that for this transformation, the column-terrace angle amounts to $53^{\circ}$. If the vantage point moves 1 unit laterally (parallel to the picture plane) from the original position, as in Figure 5C, the effect 


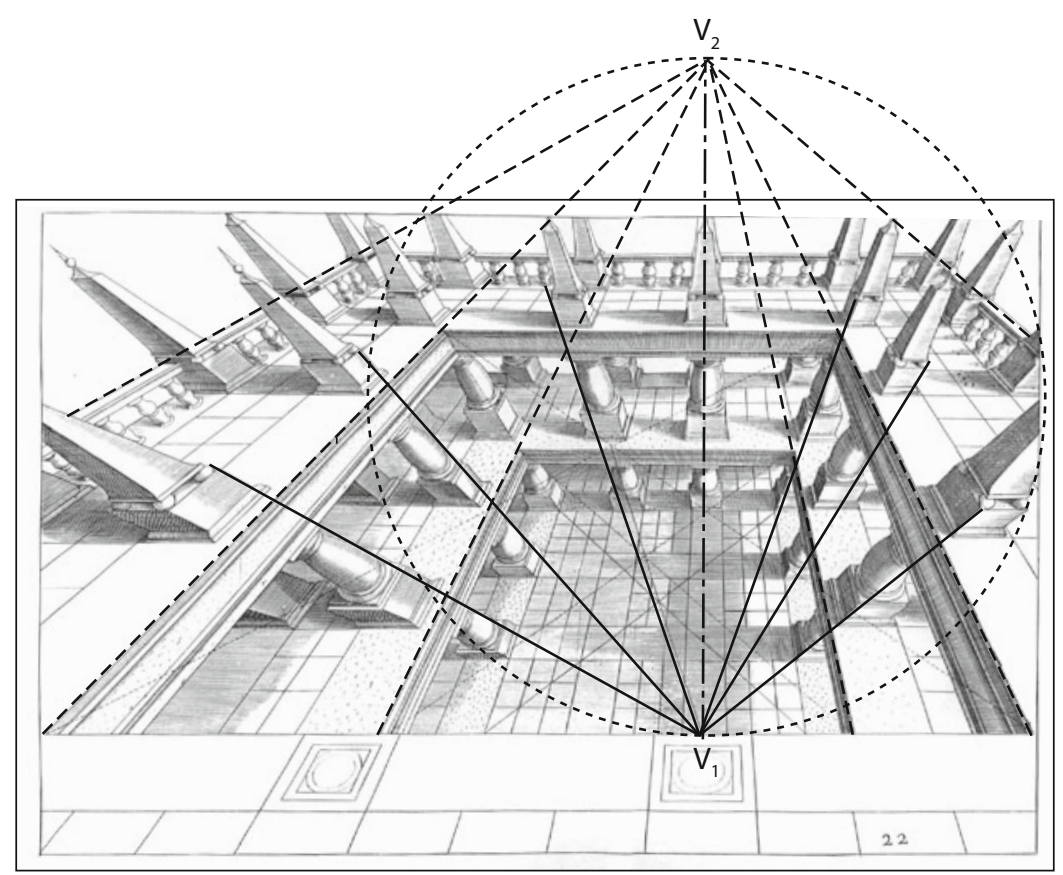

Figure 4. The perspective structure of Figure $1 . V_{1}$ is the vanishing point of the edges of the column bases (full lines), and $V_{2}$ is the vanishing points of the depth-oriented sides of the terrace (dashed lines). The two line families share a common vertical axis (dotted-dashed line). See the text for details.

is an inversely directed lateral shear of the scene, again distorting the column-terrace angle - in this case, to $63^{\circ}$. Note that these effects predicted by the geometrical analyses are consistent with the distorted appearance of Figure 1.

The effect of vantage point position was formally tested in an experiment in which Figure 1 was used as stimulus. The vantage point position theory predicts no distortions when the vantage point is positioned at the correct projection center but predicts distortions when it is positioned at some other location. The theory based on Perkins's laws predicts that the angle of Pillar 1 will look distorted because it violates these laws but does not predict that the amount of that distortion should depend on the position of the vantage point.

\section{Method}

Subjects. The subjects were 10 members of the Laboratory of Experimental Psychology at the University of Belgrade, who were not familiar with the purpose of the experiment or versed in perspective.
A

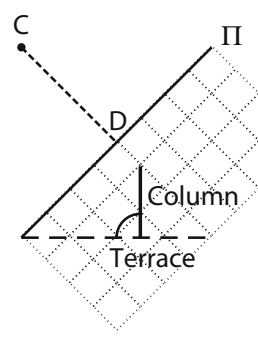

B

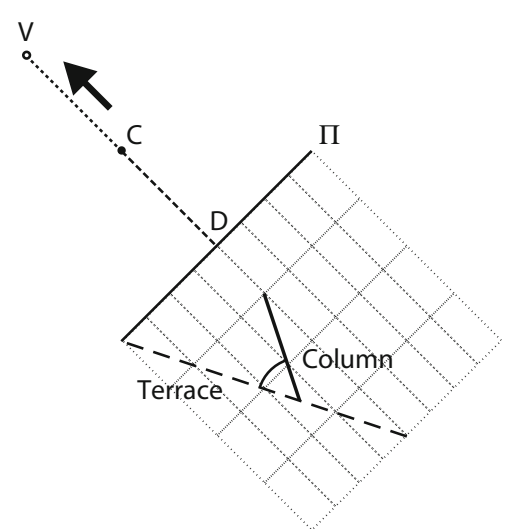

C

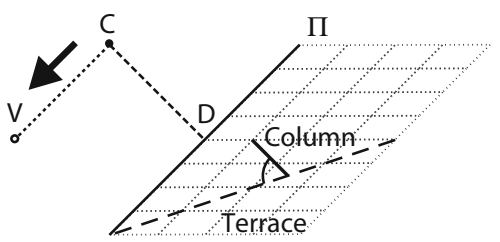

Figure 5. Effects of changes of the vantage point position on the structure of the depicted layout. The superimposed grids help illustrate the distortion effects. (A) Sketch of an initial situation with projection center $C$, projection surface $\Pi$, a terrace, and a column, subtending an angle of $90^{\circ}$ with the terrace. (B) Effect of the displacement of the vantage point $V$ orthogonally away from the projection plane. (C) Effect of the displacement of the vantage point parallel with respect to the projection plane. 


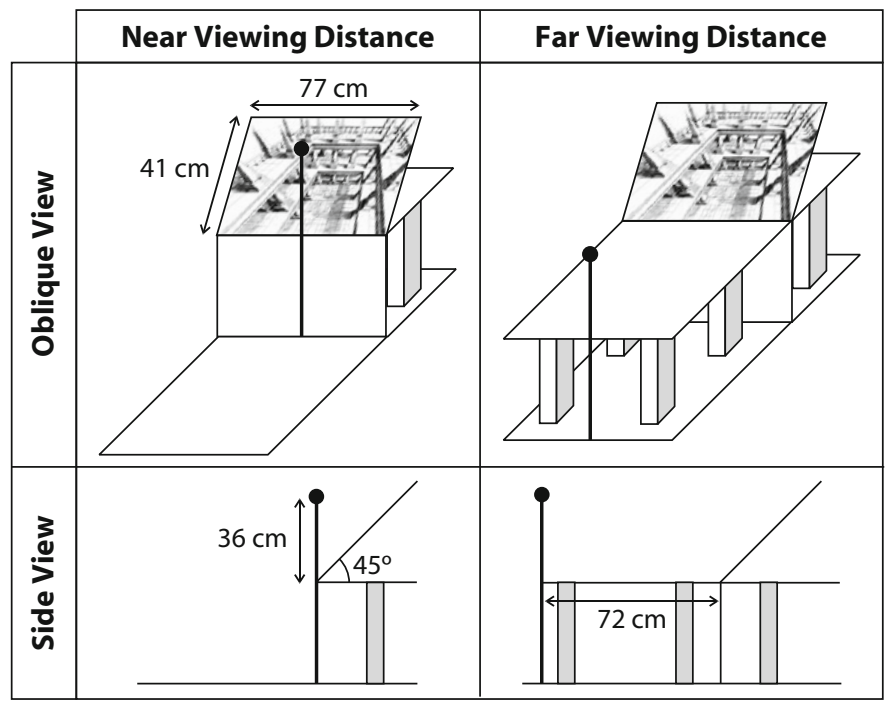

Figure 6. The setup in two conditions in Experiment 2, represented in oblique view and side view, drawn in parallel perspective. See the text for details.

Stimulus and Setup. The width $\times$ height dimensions of the display were $77 \times 41 \mathrm{~cm}$. For presentation purposes, the display was tilted back $45^{\circ}$. A horizontal stripe of the original drawing between the bottom line and V1 was cropped, so that V1 was positioned at the bottom edge of the image. There were two viewing conditions, near and far, schematically represented in Figure 6. In the near condition, the vantage point of the subject was located at the projection center associated with the drawing, as determined by the analysis above. Given the dimensions and orientation of the stimulus display, it was located $36 \mathrm{~cm}$ directly above V1. In the far condition, the vantage point was moved back horizontally for $72 \mathrm{~cm}$.

Procedure. The subjects were seated, and a headrest was used to fix the position of the vantage point. They were asked to estimate, in degrees, the angle of inclination that five selected columns (indicated by circled numerals in Figure 1) subtended with the terrace. The task was explained, with the help of a sketch similar to Figure 7, as referring to the angle that the central axis of the column subtends with its perpendicular projection upon the terrace. It was stressed that the to-be-judged angle referred to the virtual scene conveyed by the display, and not to the angle on the surface of the image. To help the judgments, to the left of the subjects at a distance of $60 \mathrm{~cm}$, there was a protractor, with angles from $0^{\circ}$ to $90^{\circ}$, indicated in steps of $5^{\circ}$; the subjects were able to see the protractor if they turned their head but could not see the display and

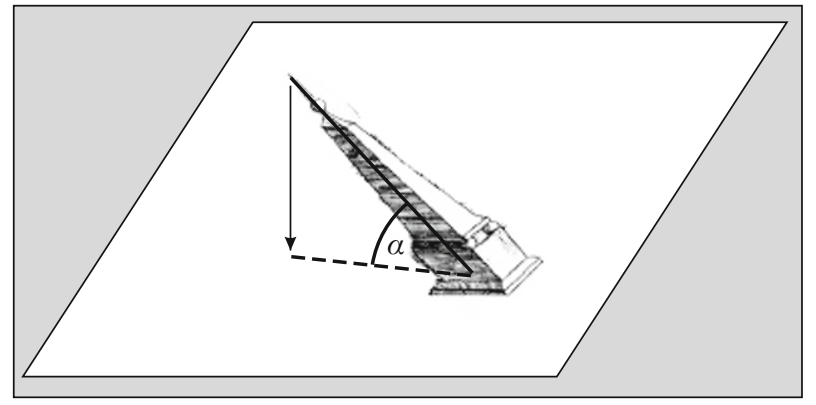

Figure 7. Scheme used to explain the task in Experiment 2 to the subjects. The task was to judge the angle subtended by the main axis of the column and its perpendicular projection upon the terrace. the protractor at the same time. The judgments were performed in pseudorandom order and were repeated four times for each column. All the subjects participated in both the near and the far conditions, half in one order, half in the other.

\section{Results and Discussion}

The results are presented in Figure 8. It can be seen that for all the columns, the judgments were closer to $90^{\circ}$ in the near condition than in the far condition, although negligibly so for column 3 . The data were analyzed with a completely repeated two-factor ANOVA, the factors being distance (near or far) and columns $(1-5)$. The distance factor was significant $\left[F(1,9)=31.57, M S_{\mathrm{e}}=2,997.6, p<.0003\right]$, the columns factor was also significant $\left[F(4,36)=34.53, M S_{\mathrm{e}}=\right.$ $1,558.8, p<.0001]$, and their interaction was significant as well $\left[F(4,36)=36.47, M S_{\mathrm{e}}=521.3, p<.0001\right]$. Planned comparisons between the far and the near conditions were significant for Columns $1[F(1,9)=38.76, p=.0002], 2$ $[F(1,9)=28.11, p=.0005], 4[F(1,9)=10.81, p=.009]$, and $5[F(1,9)=30.18, p=.0004]$, but were not significant for column $3[F(1,9)=0.10, p=.76]$.

There are three main conclusions, rather similar to the ones for Experiment 1. First, there was a clear effect of observation distance on the appearance of angular relations between elements of the depicted scene, in agreement with the vantage point position hypothesis: The angles that the columns appeared to subtend with respect to the terrace were closer to vertical in the near distance condition than in the far distance condition. The largest effect was obtained for column 1, which "straightened up" by $26^{\circ}$ from the far to the near condition.

Second, assuming that the near vantage point position corresponds to the correct projection center, according to geometrical analyses all columns should have appeared to subtend $90^{\circ}$ angles with respect to the terrace. The data showed that the average judged angle of the five columns in the near condition was $85.7^{\circ}$. Column 1 was an outlier, 


\section{Judged Angles in Experiment 2}

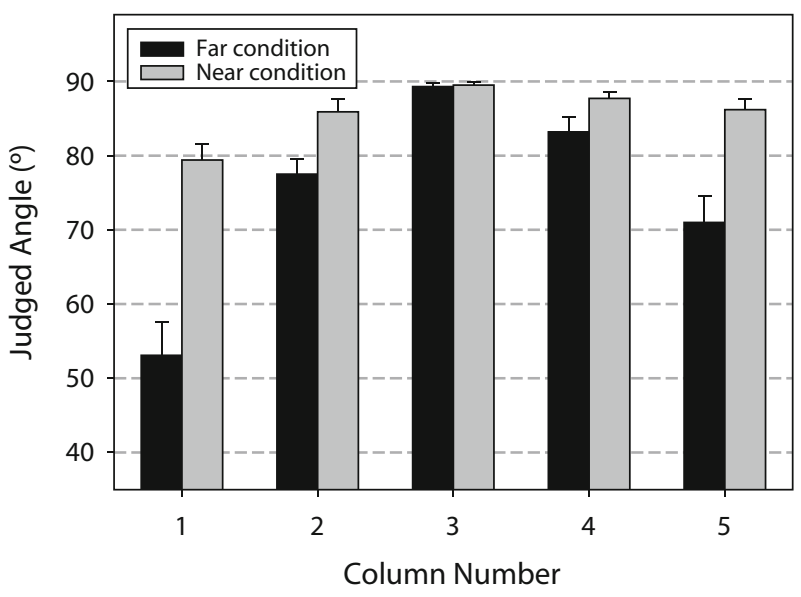

Figure 8. Mean judgments, in degrees, of angles of five columns in the far and the near vantage point conditions in Experiment 2.

with an angle of $79.4^{\circ}$; the difference from the theoretical angle of $90^{\circ}$ was significant $[t(9)=4.87, p<.0009]$. The perceived orientations of the other four columns in the near condition came rather close to the architecturally reasonable perpendicularity with respect to the terrace, probably intended by the artist.

Third, although the effect of vantage point distance on perceived orientation was in the direction prescribed by geometrical analyses, quantitatively it was distinctly smaller than predicted. The quantitative prediction for the perceived angle for the far condition can be obtained through geometrical considerations similar to those illustrated in Figure 5. Note that although the displacement of the vantage point from the near to the far condition was in the horizontal direction, it was not orthogonal to the image surface, which was tilted by $45^{\circ}$. Rather, the displacement was equivalent to a combination of an orthogonal displacement, as in Figure 5B, and a lateral displacement, as in Figure 5C. Given the dimensions and orientation of the display and setup and the positions of the two vantage points, it can be shown that both displacements had an extent of two units, with one unit being the orthogonal distance of the near vantage point from the image surface (corresponding to length CD in Figure 5). On the basis of this analysis, it can be calculated that for the far position, the predicted angle subtended by the columns and the terrace should be equal to $26.6^{\circ}$ for all columns. However, the empirically obtained judged angles in the far position ranged from $53.1^{\circ}$ for column 1 to $89.3^{\circ}$ for column 3 .

There was a close relation between the judgments for the spatial angles of the five columns with respect to the terrace in the virtual 3-D scene and their orientations in the 2-D image. Thus, for column 3 , the orientation in the image was vertical, and its judged spatial angle with respect to the terrace in the virtual scene was very close to $90^{\circ}$, for both the far and the near conditions. The absolute deviations from vertical for the other four columns in the image were, in order, $19^{\circ}$ for column $4,33^{\circ}$ for column $2,52^{\circ}$ for column 5 , and $61^{\circ}$ for column 1 . The same order applied for the judgments of their spatial angles subtended with the terrace in both conditions; that is, the farther their 2-D orientations in the image diverged from vertical, the more their perceived spatial angles with respect to the terrace in the virtual 3-D scene deviated from $90^{\circ}$. However, the 3-D judgments cannot be explained as being due simply to the corresponding 2 -D orientations. The reason is that the 3-D judgments were significantly different in the two observation conditions for all the columns (except for column 3), although their 2-D orientations were, of course, the same. Note that the instructions to the subjects, aided by the sketch in Figure 7, stressed that the to-be-judged angle was the spatial angle in the scene and not some angle in the image. Thus, although column 3 was vertical in the image, it still could have been perceived as tilted in depth with respect to the terrace, but according to the data, it was not.

The outcome of this experiment falls outside of the domain of application of the Juricevic and Kennedy (2006) theory, because their theory applies for plane shapes or plane parts of 3-D shapes (and in its current form, is developed and tested only for shapes within the ground plane) but does not, at least as yet, apply for angles between arbitrarily oriented objects within the virtual 3-D space (such as pillars and terraces), which is a more complex geometrical issue.

\section{GENERAL DISCUSSION}

Figures 1 and 2 provide examples of distorted appearance of linear perspective images: Some columns in Figure 1 appear to subtend acute angles with the terrace, and the oval pillars in Figure 2 look elliptical and, thus, violate customary architectural regularities. However, there appear to be no basic faults with de Vries's perspective constructions in either figure, nor are there good reasons to believe that he intended to depict deviant scenes (Kubovy, 1998).

Two explanations of perceived distortions were considered. The intrinsic explanation proposes that distortions will appear or not appear depending on whether projections of depicted trihedral corners violate or obey Perkins's laws. This prediction was not borne out by the data. In Experiment 1, the projection of the column base did not violate Perkins's laws, but the 3-D appearance of the column was different when the image was viewed from different distances. In Experiment 2, the base of Column 1 did violate Perkins's laws, but it exhibited quite different degrees of distortion when viewed from different distances. Note that in the formulation of Perkins's laws, only the relations of certain angles in the image are considered, and no mention is made of observation conditions; however, the data showed that the degree of distorted appearance was strongly affected by variations of viewing distance, despite the fact that such manipulations, obviously, do not change the drawings themselves. Perkins's laws may possibly be reformulated in terms of projected visual angles, rather than angles in the image, but this has apparently not been attempted yet. Nevertheless, one might argue that some observational conditions were unfavorable for the application of these laws. For example, when observed from the nearest distances, both figures subtended unusually large 
horizontal visual angles, amounting to $93.8^{\circ}$ for Figure 1 and $127.8^{\circ}$ for Figure 2; for comparison, a photograph $20 \mathrm{~cm}$ across observed from a distance of $50 \mathrm{~cm}$ subtends $22.6^{\circ}$, the same as a painting $1 \mathrm{~m}$ across observed from 2.5 m. However, Perkins's laws do not refer to whole pictures but to their local features involving projections of trihedral angles, which subtend fractions of the visual angle of the whole picture. It is not unusual for museum visitors to move closer to large-scale paintings in order to better inspect some details. Thus, one might have expected that for Figure 1, the violation of Perkins's laws would have been more obvious to viewers in the near than in the far observation condition. However, the outcome was the opposite, in that the perceived orientation of Pillar 1 deviated much less from $90^{\circ}$ in the near than in the far condition.

The technical and artistic perspective literature cautions against using excessive sizes of visual angles, because they are known to lead to distorted appearance. For example, Leonardo da Vinci gave corresponding practical advice for painters on how to avoid such unwanted effects, by using smaller angles (Richter, 1970). Olmer (1943/1949) favored the use of horizontal visual angles up to $37^{\circ}$, and presented examples with drawings of cubes that generally appeared correct if confined within the central $37^{\circ}$, but looked distorted if drawn outside these limits. Kubovy (1986) pointed out that, in the first case, the drawings of cubes tended to conform to Perkins's laws and, in the second case, tended to violate them. Thus, these laws seem to be useful diagnostic indicators of peripherally located projected cuboids and trihedral right angles. However, otherwise, they have limited generality for the explanation of perceptually inadequate depictions in geometrically correct linear perspective images. For example, they cannot be applied directly to the case of the elongated oval columns in Figure 2. Furthermore, both the low cuboid bases of the oval columns and the high cuboid bases of the pyramidal columns in this figure do adhere to Perkins's laws. Their elongated appearance, as measured in Experiment 1, does not violate architectural conventions and, therefore, is not immediately seen as a distortion, yet it inadequately conveys the intended spatial features of the depicted objects.

The extrinsic explanation claims that the distorted appearance of these images can be attributed to the vantage points from which they are usually observed and that they should not look distorted if observed from the positions of their projection centers. To test this idea, it was necessary to reconstruct these positions. Given some assumptions, it was possible to do that and to establish that their distances from the two images amount to fractions of the image widths. Such distances are much too close for comfortable viewing, and the visually acceptable vantage points are located significantly farther from the images, away from projection centers. Enlarging the images increased the distances of the respective projection centers and made it possible for observers to adopt the projectively appropriate vantage point locations. In general accord with the vantage point hypothesis, from such observation positions, the perceived layouts looked much less distorted and appeared much closer to the scenes that de Vries probably meant to portray than when observed from more distant vantage points.
In sum, together with previous data, the results of the present study strongly argue that for linear perspective images to represent 3-D scenes to their observers as faithfully as 2-D figures possibly can, it is not enough that their construction is geometrically correct. In addition, in accord with recommendations from artistic and technical practice (Olmer, 1943/1949; Richter, 1970), such images should not involve scenes with excessively large visual angles, especially if the formats in which they are subsequently presented preclude the viewers from occupying vantage points reasonably close to the projection center. If such prescriptions are not followed, more or less obvious deviations of the perceived scenes from the intended scenes may result, as demonstrated here by the two de Vries drawings.

One problem for the vantage point hypothesis is that although the data were in qualitative accord with it, in that further observation distances were associated with stronger distortions, the quantitative predictions were off the mark, in that the effects obtained, although salient, were appreciably smaller than predicted. In Experiment 1, the distances required to perceive the ordained elongation ratios of the column base were several times larger than predicted. In Experiment 2, in the far condition, the perceived angles of the pillars were not as deviant from $90^{\circ}$ as predicted, and the angles were also not all the same, as predicted, but different for different columns; furthermore, in the near condition, the perceived angle of Pillar 1 was smaller than the predicted $90^{\circ}$.

A possible reason for these discrepancies of the obtained results from the predictions is the well-known dual nature of pictures: The stimulus displays were 2-D images that represent 3-D scenes with conflicting depth information. On the one hand, pictorial cues in these displays, such as linear perspective, texture gradients, interposition, and height in the field, provided information about depth. On the other hand, whether observed from correct or incorrect vantage points, cues from stereopsis, parallax, accommodation, and convergence clearly indicated to viewers that the displays were flat surfaces. Detailed analyses of depth cues in paintings go back at least to Ames (1925) and Schlosberg (1941). Sedgwick (1993) suggested that the depth impression elicited by 2-D images may be based on a compromise between the different depth cues. In particular, the conflict between the depth cues may have diminished the extent of depth elongation prescribed by pure linear perspective criteria. Flatness may also have increased the salience of the 2-D properties of the image, relative to the corresponding 3-D features. For example, although perspective cues indicated that the columns in Figure 1 were all parallel in the conveyed virtual scene, in the image they were all differently slanted (the particular values of slants are noted above), which may have contributed to the fact that, in the percept, they did not appear parallel but somewhat converging into depth. In Experiment 1, the flatness information may have been enhanced by motion parallax information due to the movements of the observers, thus possibly contributing to the much smaller than expected distance effects. On the other hand, when the images were in close view, 3-D impressions may have been enhanced by the 
large visual angle and diminished salience of the more peripherally located picture frames. A number of studies, reviewed by Rogers (1995), have shown compression of perceived depth in images of spatial scenes, although different studies have reported different degrees of compression. More recent experiments have confirmed these effects (Juricevic \& Kennedy, 2006; Saunders \& Backus, 2006; Todorović, 2008). To what extent the amount of depth compression in images can quantitatively predict the extent of distortions such as those studied in this article remains an important topic for further research.

\section{AUTHOR NOTE}

This research was supported by Grant 149039D from the Serbian Ministry of Science. Correspondence concerning this article should be addressed to D. Todorović, Laboratory of Experimental Psychology, Department of Psychology, University of Belgrade, Čika Ljubina 18-20, 11000 Belgrade, Serbia (e-mail: dtodorov@f.bg.ac.rs).

Note-Accepted by the previous editorial team, when Thomas H. Carr was Editor.

\section{REFERENCES}

AMES, A., JR. (1925). The illusion of depth from single pictures. Journal of the Optical Society of America, 10, 137-148.

CutTing, J. E. (1988). Affine distortions of pictorial space: Some predictions for Goldstein (1987) that La Gournerie (1859) might have made. Journal of Experimental Psychology: Human Perception \& Performance, 14, 305-311.

Cutting, J. E. (1993). On the efficacy of cinema, or what the visual system did not evolve to do. In S. R. Ellis (Ed.), Pictorial communication in virtual and real environments (2nd ed., pp. 486-495). London: Taylor \& Francis.

DE LA Gournerie, J. (1859). Traité de perspective linéaire. Paris: Dalmont et Dunod.

Deregowski, J. B., \& PARKer, D. M. (1992). Three-space inference from two-space simulation. Perception \& Psychophysics, 51, 397-403.

DE VRIES, J. V. (1970). Perspective. New York: Dover. (Original work published in 1604)

Dubery, F., \& Willats, J. (1983). Perspective and other drawing systems. London: Herbert.

FIELD, J. V. (1986). Piero della Francesca's treatment of edge distortion. Journal of the Warburg \& Courtauld Institutes, 49, 66-90.

FIELD, J. V. (1997). The invention of infinity: Mathematics and art in the Renaissance. Oxford: Oxford University Press.

Goldstein, E. B. (1979). Rotation of objects in pictures viewed at an angle: Evidence for different properties of two types of pictorial space. Journal of Experimental Psychology: Human Perception \& Performance, 5, 78-87.

Goldstein, E. B. (2001). Pictorial perception and art. In E. B. Goldstein (Ed.), Blackwell handbook of perception (pp. 344-378). Oxford: Blackwell.

Goodman, N. (1976). Languages of art: An approach to a theory of symbols. Indianapolis: Hackett.

GreEN, R. (1983). Determining the preferred viewpoint in linear perspective. Leonardo, 16, 97-102.

HABER, R. N. (1980). Perceiving space from pictures: A theoretical analysis. In M. A. Hagen (Ed.), What then are pictures? The psychology of representational art (pp. 3-31). New York: Academic Press.

Halloran, T. O. (1993). The frame turns also: Factors in differential rotation in pictures. Perception \& Psychophysics, 54, 496-508.

HaLlORAN, T. O. (2006). Finding a picture's station point: Reconstructing virtual space when the usual way will not work. Behavior Research Methods, 38, 107-116.

JuRICEVIC, I., \& KENNEDY, J. M. (2006). Looking at perspective pictures from too far, too close, and just right. Journal of Experimental Psychology: General, 135, 448-461.

KemP, M. (1990). The science of art: Optical themes in Western art from Brunelleschi to Seurat. New Haven, CT: Yale University Press.
Kubovy, M. (1986). The psychology of perspective and Renaissance art. Cambridge: Cambridge University Press.

Kubovy, M. (1998). Psychology of perspective. In M. Kelly (Ed.), Encyclopedia of aesthetics (Vol. 3, pp. 481-485). New York: Oxford University Press.

Nicholls, A. L., \& Kennedy, J. M. (1993). Angular subtense effects on perception of polar and parallel projections of cubes. Perception \& Psychophysics, 54, 763-772.

Olmer, P. (1949). Perspective artistique (Vols. 1 and 2). Paris: Plon. (Original work published 1943)

PERKINS, D. N. (1973). Compensating for distortion in viewing pictures obliquely. Perception \& Psychophysics, 14, 13-18.

Pirenne, M. H. (1970). Optics, painting and photography. Cambridge: Cambridge University Press.

RichteR, J. P. (1970). The notebooks of Leonardo da Vinci. New York: Dover.

Rogers, S. (1995). Perceiving pictorial space. In W. Epstein \& S. Rogers (Eds.), Perception of space and motion: Handbook of perception and cognition (2nd ed., pp. 119-163). San Diego: Academic Press.

SAUnders, J. A., \& BACKUS, B. T. (2006). The accuracy and reliability of perceived depth from linear perspective as a function of image size. Journal of Vision, 6, 933-954.

SCHLOSBERG, H. (1941). Stereoscopic depth from single pictures. American Journal of Psychology, 54, 601-605.

SeDGWICK, H. A. (1980). The geometry of spatial layout in pictorial representation. In M. A. Hagen (Ed.), The perception of pictures: Vol. I. Alberti s window: The projective model of pictorial information (pp. 34-90). New York: Academic Press.

SEDGWICK, H. A. (1993). The effects of viewpoint on the virtual space of pictures. In S. R. Ellis (Ed.), Pictorial communication in virtual and real environments (2nd ed., pp. 460-479). London: Taylor \& Francis.

SHEPARD, R. N. (1981). Psychophysical complementarity. In M. Kubovy \& J. R. Pomerantz (Eds.), Perceptual organization (pp. 279-341). Hillsdale, NJ: Erlbaum.

SHEPARD, R. N. (1992). Mind sights. New York: Freeman.

ten Doesschate, G. (1964). Perspective: Fundamentals, controversials, history. Amsterdam: Nieuwkoop B. de Graaf.

ToDorović, D. (2005). Geometric and perceptual effects of the location of the observer vantage point for linear-perspective images. Perception, 34, 521-544.

ToDOrović, D. (2008). Is pictorial perception robust? The effect of the observer vantage point on the perceived depth structure of linear perspective images. Perception, 37, 106-125.

Veltman, K. H. (1986). Studies on Leonardo da Vinci: I. Linear perspective and the visual dimensions of science and art. Munich: Deutscher Kunstverlag.

Vishwanath, D., Girshick, A. R., \& Banks, M. S. (2005). Why pictures look right when viewed from the wrong place. Nature Neuroscience, 8, 1401-1410.

Wallach, H. (1976). The apparent rotation of pictorial scenes. In M. Henle (Ed.), Vision and artifact (pp. 65-69). New York: Springer.

Wittkower, R., \& Carter, B. A. R. (1953). The perspective of Piero della Francesca's "Flagellation." Journal of the Warburg \& Courtauld Institutes, 16, 292-302.

YANG, T., \& KUBOVY, M. (1999). Weakening the robustness of perspective: Evidence for a modified theory of compensation in picture perception. Perception \& Psychophysics, 61, 456-467.

\section{NOTES}

1. As an example of possible neglect of perspective laws in this drawing, note that the objects on top of the oval columns have circular shapes, although they were probably meant to represent projections of spheres and, in that case, should have been drawn as ellipses, just as in Raphael's School of Athens (see Todorović, 2005).

2 . These findings suggest that it might be of interest to examine the attainability of projection centers for many other artworks. A rare case for which this issue was studied is Leonardo's famous Last Supper, whose correct vantage point is located several meters above the floor and, thus, is unattainable for regular visitors of the painting (see Kubovy, 1986). 